\title{
MODELLING OF COMPLEX SYSTEMS BY MEANS OF PARTIAL ALGEBRAS
}

\author{
Jiri Bila ${ }^{1, \otimes}$, Ricardo Rodríguez Jorge ${ }^{2}$, Martin Novak ${ }^{1}$ \\ ${ }^{1}$ Institute of Instrumentation and Control Engineering, Czech Technical University in Prague, Czech Republic \\ ${ }^{2}$ Department of Mechatronics, Technological University of Ciudad Juarez, Mexico \\ jiri.bila@fs.cvut.cz ${ }^{\boxplus}$, ricardo_rodriguez@utcj.edu.mx
}

\begin{abstract}
Complex systems are very hard to describe by some unified language and calculus. In cases when their nature is very heterogeneous is possible to use with advantage state description. Formalization of operations on the set of states usually leads to partial algebras. The work with partial algebras is rather difficult and unpractical. From this reason some methods approximating partial algebras by some more symmetrical objects are searched for. In this paper there is proposed an approximation of this algebras by free cyclic groups. Then using the combination of Matroid Theory and Ramsey theory of graph the prediction of a possible appearance of emergent situation is executed. Data and knowledge used in the paper for the demonstration of developed method application are from the field of Ecology.
\end{abstract}

Keywords: partial algebras, cyclic groups, qualitative distance, matroid, matroid bases, prediction of emergent situation on complex system.

Published: 24 June 2019

\section{Introduction}

We will deal with complex systems that can be described by a set of states and transitions (transformations) between states. The description of states is not absolute, it is given by an expert and the transformation of states and trajectories created by transformations symbolize the complex behavior of the system, which actually takes place under the level of symbolism of transformations and states.

Just as transformations are not defined for all states, operation for composition of transformation is not allowed for all transformations. So, we are talking about a complex system that is described by partial algebras of transformations.

The mathematical issue of partial algebras has been discussed many times in legendary books and therefore it is not necessary to develop a basic theory in this article. Rather, we will focus on some of the challenges that arise when trying to apply partial algebra theory to problems arising in complex systems.

We will address the following tasks:

- $\quad$ The task of reachability of states (i.e. - from which states is possible to reach which states)

- $\quad$ The task of approximating sub algebra of a partial algebra by a cyclic group of transformations.

- The task of predicting an emergent situation on complex system using cyclic group of transformations in the role of chronometer.

For the first two tasks, algebra is sufficient, for the third task we use the method developed and verified, e.g., in $[2,6,7]$ that uses Theory of Matroids, Ramsey Theory of Graph and an emergent calculus working with power and complexity of an emergent phenomenon.

An indispensable phase of a complex system state description is the selection and formation of a set of states. (It will be explained in Section 4.)

\section{Related Works}

A great problem of this paper is its interdisciplinarity. In the next small overview are introduced only some works that could serve as an information how we approached the solved problem. Field C. is related to application area of this paper - Eco complex systems.

A. Complex systems and emergent situations. The basic concept of complex system does not differ from the concept of the system. Set of elements, bonds between them and mutual interactions. However, the deeper analysis of complex systems discovered some peculiar properties that are very substantial for their identification 
and control (e.g., quasi-stability in state changes. nonlinear characteristics, self-organizing processes, etc.). The theory of complex systems is introduced, e.g., in [1], [6], [8]. Emergent and self-organizing phenomena are dealt with in [6], [16].

B. State description and algebraic characteristics of state transformations. The fundamental operations in the state description of complex systems are the selection of states and the construction of state transformations.

There are a number of methods for the selection of quantitative states. (We introduce here only the Principal Component Analysis [4] and its use in work [3]). These methods generally lead to a reduction of the original set of states. Significantly different is the situation when selection of states is done by expert [2]. After the constitution of sets of states and transformations follows algebraic description. The most accurate description of the algebraic side of transformations is the description by means of partial algebras, which is of course the most sophisticated. Of the many works we only mention [9 -10].

C. Principles of modeling of evolution for complex and Eco complex systems. The application of the methods proposed in this article will be illustrated in the field of ecology. We are particularly interested in the issue of global climate change. From this area we present relevant subject background in works [17-19].

\section{Preliminaries}

General concepts

We will work with the state description of the system. Transformations occur on state space as transitions between states.

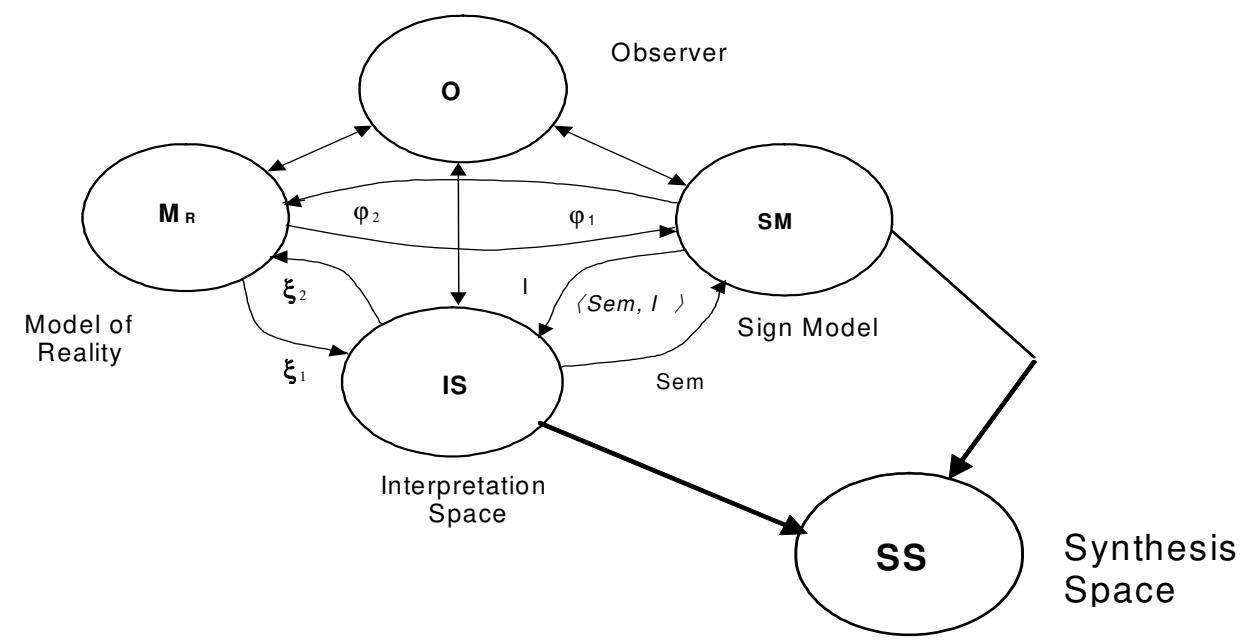

Figure 1: Relations between Model of Reality, Sign Model, Interpretation Space and Synthesis Space

The most general idea of the relationship between Model of Reality, Sign Model, Interpretation Space and Synthesis Space is in Figure 1. Figure 1 has already been mentioned in our recent papers. Here we will recall some basic concepts:

Model of Reality (MR) is a cognitive reflection of reality in our mind.

Sign Model (SM) of the system is a representation of the system by structure of signs. For technological and mathematical fields are nearest signs symbols.

Interpretation space (IS) is a collection of knowledge that contains semantic contents of signs and symbols.

Interpretation of signs and sign structures is considered as a mapping from SM to IS.

Compartment on a system is a part of the system - in which is possible to form local sub model of the system model and that is considered as a representative for investigation of phenomena on the system and in our case especially of emergent phenomena and emergent situations.

In Synthesis Space (SS) - some not prepared solutions are discovered and also emergent situations are predicted (detected).

In our case, the formation of a set of states is done by an expert using SM and IS. In SM there are signs and constructions, in IS there is the meaning of symbols and a description of what is under the states and their transformations. What is the main motive of the article are algebras in SM.

We consider partial algebras

$$
\mathbf{A}=\langle X, o, D\rangle
$$


where $X$ are the elements, $o$ is a compositional operation, and $D$ is a description of the conditions under which the $o$ operation can be applied to compose the elements of $X$.

$X$ are transformations based on the state description of the complex system.

$$
S M=\langle S, \boldsymbol{A}\rangle=\langle S,\langle X, o, D\rangle\rangle,
$$

where $S M$ is a Sign Model, $S$ is a set of states and $X$ is a set of transformations.

For unary transformations hold:

$$
T_{1} \in X, T_{1}\left(S_{i}\right)=S_{j} \text {, for some } S_{i}, S_{j} \in S \text {, for which hold conditions } D \text { of application } T_{1}
$$

The continuation from state $S_{j}$ by some $T_{2}$ transformation is understood as composition of $T_{2}$ with $T_{1}$. However, such a transformation may not exist. The composition operation $o$ is generally neither transitive nor commutative, nor associative. (More in detail in Section 6.)

Partial algebra is very difficult to work with. If we want to solve the classical task of reaching a state based on some other state, we must examine each application of the operation $o$ before its use according to the conditions specified in $D$.

\section{Matroid Theory and Ramsey Theory of Graph}

The concept of this synthesis and its application in real examples have been introduced, e.g., in [6-7]. We introduce here only a basic knowledge.

a) Extension of a matroid base is possible to do only from the outside the matroid and it is considered as an indicator (initiator) of an emergent phenomenon (Emergent Situation - EMS).

b) Matroid is usually introduced [13] as the following structure

$$
M=\left\langle X, I N D,\left\{N_{1}, N_{2}, \ldots, N_{n}\right\}\right\rangle=\langle X, M B\rangle,
$$

where $X$ is the ground set of elements (components), IND is a relation of independence, $N_{1}, N_{2}, \ldots, N_{n}$ are independent sets and $\boldsymbol{M B}$ is a set of matroid bases.

c) Matroid bases are maximum (according to cardinality) independent sets. With advantage is used in practical cases relation Dependence $(\boldsymbol{D N T})$.

d) One of Dependence binary relations relativized to some problem factor $\alpha-\left(\boldsymbol{D N} \boldsymbol{T}\left(x_{1}, x_{2} \mid \alpha\right)\right)$ - has been defined in [6-7].

e) Relation $\left(\boldsymbol{D} \boldsymbol{N} \boldsymbol{T}\left(x_{1}, x_{2} \mid \alpha\right)\right)$ has been discovered as a relation equivalence.

f) For $\left(\boldsymbol{D N} \boldsymbol{T}\left(x_{1}, x_{2} \mid \alpha\right)\right)$ holds simple theorem:

\section{Theorem 1:}

$$
\forall x_{-} 1, x_{-} 2 \in X,\left(\operatorname{Not}\left(\boldsymbol{D N} \boldsymbol{T}\left(x_{1}, x_{2} \mid \alpha\right)\right) \Leftrightarrow\left(\boldsymbol{I N} \boldsymbol{D}\left(x_{1}, x_{2} \mid \alpha\right)\right) .\right.
$$

For relation $\boldsymbol{I N D}(5)$ is possible to use the following consequences:

The bases $(\boldsymbol{M B})$ are constructed as perfect sub-graphs in a perfect graph $G_{p}$ (each node is connected with all other nodes of $X$ ). The independent and dependent elements in the perfect graph are easy constructed by coloring the edges of this complete graph $G_{p}$ by two colors and the formalism of Ramsey numbers $R(\# \boldsymbol{B}, Y)), \boldsymbol{B} \in \boldsymbol{M} \boldsymbol{B}$ is offered to be used. We introduce now a free formulation of relations on a perfect graph $G_{p}$ colored by two colors as a consequence of famous Ramsey theorem [14, 15]:

Theorem 2: A perfect graph $G_{p}=(V, E)$ with $\mathrm{n}$ nodes where each edge belongs to class A or to class B contains a complete subgraph with a nodes connected by edges from class A or a complete subgraph with $b$ nodes connected by edges from class B.

Such a number $R(a, b)=n$ is called Ramsey number.

\section{Cyclic groups}

From the theory of cyclic groups we use the following theorems (we do not prove them because their proofs are known from the literature):

V1: Each cyclic group is commutative.

V2: Each subgroup of a cyclic group is cyclic.

V3: Let's have $k$ free cyclic groups $G_{1}, G_{2}, \ldots, G_{k}$.

The group, whose elements are constructed as elements of the direct product of the given cyclic groups, is cyclic.

Another explanation for this theorem is in Section 6 . 


\section{Emergent calculus [6-7]}

The calculus for the emergent situation in a complex system (that is introduced in the paper) associates two variables for emergent situation - The power $H_{P}$ of emergent phenomenon (that is usually computed by quantities of symptoms) and the complexity $H_{C O M}$ of emergent phenomenon (that is associated with action activities sometimes called drivers).

Essential relations between the power $H_{P}$ and the complexity $H_{C O M}$ are expressed by two equations

$$
\begin{aligned}
& H_{p}(\boldsymbol{B}+\mathbf{1})=H_{p}(\boldsymbol{B})+(u / c) H_{C O M}(\boldsymbol{B}), \\
& H_{C O M}(\boldsymbol{B}+\mathbf{1})=H_{C O M}(\boldsymbol{B})+u H_{p}(\boldsymbol{B}),
\end{aligned}
$$

where $\boldsymbol{B} \in \boldsymbol{M} \boldsymbol{B}$ is a basis of a matroid and $\boldsymbol{B}+\mathbf{1}$ is the basis $\boldsymbol{B}$ extended by one element. Variables $H_{p}(\boldsymbol{B})$, $H_{p}(\boldsymbol{B}+\mathbf{1})$ and $H_{C O M}(\boldsymbol{B}), H_{C O M}(\boldsymbol{B}+\mathbf{1})$ are power and complexity of emergent phenomenon related to bases $\boldsymbol{B}$ and $\boldsymbol{B}+\mathbf{1}$.

Symbol $u$ denotes the quotient of self organization and $c$ is the limit of this quotient (the best self organization). Quotient $(u / c) \in\langle 0,1\rangle$ represents "intelligence" of self organizing process that will execute the emergent phenomenon. Operating with equation (6) we obtain contribution to power released by emergent phenomenon

$$
\Delta H_{p}(\boldsymbol{B}+\mathbf{1})=(u / c) H_{C O M}(\boldsymbol{B}) .
$$

The contribution to Power of the emergent phenomenon $\Delta H_{p}(\boldsymbol{B}+\mathbf{1})$ that has an intuitive meaning (e.g., damage of houses by floods) in some level of the description is estimated by quantities of external variables (symptoms) $s_{i}, i=1, \ldots, n$ for emergent $\left(s_{i e m}\right)$ and for nominal $\left(s_{\text {inom }}\right)$ situations:

$$
\Delta H_{p}(\boldsymbol{B}+\mathbf{1})=\sum_{i=1}^{n}\left(\omega_{i}\left(s_{i e m} / s_{\text {inom }}\right)^{2}\right)^{1 / 2}, \quad \text { for } i=1, \ldots, n,
$$

where $\omega_{i}$ are quotients of importance. The contribution to power of emergent phenomenon results in a dimensionless real number expressed here in \% (for example, contribution for $20 \%$ is calculated as $(120 / 100)=1.2) . H_{C O M}(\cdot)$ in equation is approximated in our case by number of elements of basis $\boldsymbol{B}$, i.e. \# $\boldsymbol{B}$.

In order to use expression (8) for computation of the number of matroid basis is necessary to set up the quotient $(u / c)$. In works [6-7] were introduced very rough quotients $(u / c)$.

A basic rule for detecting an emergent situation is as follows:

$$
I F(\# E \geq \min \Delta f(R N)) \Rightarrow(P A E S)
$$

where $E$ is a set that extends the matroid $\langle X, \boldsymbol{M B}\rangle$ (expression (4)) and contains at least one element $e$ extending basis $\boldsymbol{B}$. The number min $\Delta f(R N)$ is the minimal difference between the next Ramsey number and the current Ramsey number, and PAES denotes the "Possible Appearance of an Emergent Situation".

\section{Selection of States for the Description of Complex Systems}

As we have dealt with in many places, e.g., in [2-3], the selection of states for complex system description is essential and most important. We describe $M R$ using $S M$ with interpretation in $I S$. An obvious condition for selecting "correct" states is their relevance to the behavior of the described system. While in the work [2] we were based on the expert qualitative assignment of states and using the Saaty method [12], we determined the importance of individual states, in the work [3] we used the standard Principal Component Analysis for extraction of symptoms (states) [4]. In both cases, this is a mapping between $S M$ and $I S$ - Figure 1.While in the case of a qualitative description correspond to the signs denoting the states the knowledge structures (e.g., the term "Low Humidity" corresponds in the $I S$ to structure: how measured, how it is built, what units it has, where it is measured, what is its scale, how it relates to other states) ), in the case of quantitative states, we generally find a vector of variables that have values on the scale of real numbers.

Both methods (Expert method with Saaty calculus and Principal Component Analysis) lead to calculations of eigenvalues (eigenvalue vectors) from matrices of "estimates". If there are more states (there are 19 in the example in Section 5), the prioritization of states is difficult. Especially for this reason, it is not possible to model a complex system of hundreds of states as a whole and usually is used the decomposition of complex system to subsystems, depending on the task.

\section{State Transformations}

In our case the states are given and the set of states is finite. Transformations are given as transitions (allowed, not allowed) between states. 
So, it is not about constructing "new" states from "old" states, or about synthesizing states using fixed structures. This separates us from the field of production systems, formal grammars and abstract automata as parsers. These resources are not actual in real situations in complex systems.

Consider a sequence of transformations (from Table 1)

$$
\begin{gathered}
\mathrm{S} 2 \rightarrow \mathrm{S} 3 \rightarrow \mathrm{S} 15 \rightarrow \mathrm{S} 8 \rightarrow \mathrm{S} 17 \\
T 1: \mathrm{S} 2 \rightarrow \mathrm{S} 3, \text { T2: } \mathrm{S} 3 \rightarrow \mathrm{S} 15, \text { T3: } \mathrm{S} 15 \rightarrow \mathrm{S} 8, \text { T4: S8 } \rightarrow \mathrm{S} 17 .
\end{gathered}
$$

We can easily find (from Table 1) that composition $T 4$ o T3 o T2 oT1 is not real because at least two transformations do not exist.

Example: Table 1 corresponds to following states: S1...Low local humidity, S2... Medium local humidity, S3...High local humidity, S4...Local fog, S5...Regional fog (covering an area greater than 20 km2), S6...High volume of water absorbed in the soil, S7...Local floods, S8...Decrease of volume of ground water, S9...Rain, S10...Snow, S11...Longtime local dry atmosphere (arid soil), S12...Semi-clear weather, S13... Very cloudy weather and overcast, S14...Dense clouds "prepared" for raining, S15...Strong wind, S16...Storm, S17...High evapotranspiration, S18...Low evapotranspiration, S19...Drought.

These are states describing the Eco complex system given by the expert. (There is no point in asking, for example, "Where is low local humidity." The expert imagines what it means, how to measure it, what the surrounding situation is at low humidity, etc.).

From this point of view, it is also necessary to understand the transformation table - Table 1 . Though the symbolism in Table 1 is very simple - " 1 " transformation possible, " 0 " transformation impossible, "*" irrelevant question), the case of irrelevant question is important from the point of view of composition operation $o$. This is, e.g., the case where the composition of transformations is forbidden.

Table 1. Matrix of transformations in the considered ecosystem.

\begin{tabular}{|c|c|c|c|c|c|c|c|c|c|c|c|c|c|c|c|c|c|c|c|}
\hline & S1 & S2 & S3 & S4 & S5 & S6 & S7 & S8 & S9 & S10 & S11 & S12 & S13 & S14 & S15 & S16 & S17 & S18 & S19 \\
\hline & 1 & 1 & 0 & 0 & 0 & 0 & $*$ & $*$ & $*$ & $*$ & 1 & $*$ & $*$ & $*$ & $*$ & 1 & 1 & 1 & 1 \\
\hline S2 & 0 & 1 & 1 & 1 & 0 & 0 & $*$ & 0 & 1 & 0 & 0 & 0 & 1 & 1 & 0 & 0 & 1 & 1 & 0 \\
\hline S3 & 0 & 1 & 1 & 1 & 1 & 1 & 1 & 0 & $*$ & $*$ & 0 & $*$ & $*$ & $*$ & $*$ & 1 & 1 & 0 & 0 \\
\hline S4 & 0 & 0 & 1 & 1 & 1 & $*$ & $*$ & $*$ & 1 & 1 & 0 & 1 & 1 & $*$ & 0 & 0 & 1 & 1 & 0 \\
\hline S5 & 0 & 1 & 1 & 1 & 1 & $*$ & $*$ & 0 & 1 & 1 & 0 & 1 & 1 & $*$ & 1 & 0 & 0 & 1 & 0 \\
\hline S6 & 0 & 1 & 1 & 1 & 0 & 1 & 1 & 0 & $*$ & $*$ & 0 & 1 & 1 & $*$ & $*$ & 0 & 1 & 1 & 0 \\
\hline S7 & 0 & 1 & 1 & 1 & 0 & 1 & 1 & 0 & 1 & 0 & 0 & 1 & 1 & 1 & 1 & 0 & 0 & 1 & 0 \\
\hline S8 & 1 & 0 & 0 & 0 & 0 & 0 & 1 & 1 & 0 & 0 & 1 & 0 & 0 & 1 & 0 & 1 & 0 & 0 & 1 \\
\hline S9 & 0 & 1 & 1 & 1 & 1 & 1 & 1 & 0 & 1 & 1 & 0 & 1 & 0 & 1 & 1 & 1 & 0 & 1 & 0 \\
\hline S10 & 0 & 0 & 0 & 0 & 0 & 1 & 1 & 0 & 1 & 1 & 0 & 1 & 1 & 0 & 0 & 0 & 0 & 1 & 0 \\
\hline S11 & 1 & 0 & 0 & 0 & 0 & 0 & 0 & 1 & 0 & 0 & 1 & 0 & 1 & 0 & 0 & 0 & 0 & 0 & 1 \\
\hline S12 & $*$ & $*$ & $*$ & 0 & 0 & 1 & $*$ & $*$ & 1 & 1 & 0 & 1 & 1 & 0 & 0 & 0 & 1 & 1 & 0 \\
\hline S13 & $*$ & $*$ & $*$ & 0 & 0 & $*$ & $*$ & 0 & 0 & 0 & 0 & 1 & 1 & 1 & $*$ & 0 & 1 & 1 & 0 \\
\hline S14 & $*$ & $*$ & $*$ & 0 & 0 & $*$ & $*$ & $*$ & 1 & $*$ & 0 & 1 & 1 & 1 & 1 & 1 & 0 & 0 & 0 \\
\hline S15 & 0 & 0 & 1 & 1 & 0 & 0 & 1 & 0 & 1 & 0 & 0 & 0 & 0 & 0 & 1 & 0 & 0 & 1 & 0 \\
\hline S16 & 1 & 1 & 0 & 0 & 0 & 0 & 0 & 1 & 0 & 0 & 1 & 1 & 0 & $*$ & 1 & 1 & 1 & 0 & 1 \\
\hline S17 & 1 & 1 & 0 & 1 & 1 & 1 & 0 & 0 & 1 & 1 & 0 & 1 & 0 & $*$ & 1 & 0 & 1 & 1 & 0 \\
\hline S18 & 1 & 0 & 0 & 0 & 0 & 0 & $*$ & 1 & 0 & 0 & 1 & 1 & 0 & $*$ & 1 & 0 & 0 & 1 & 0 \\
\hline S19 & 1 & 0 & 0 & 0 & 0 & 0 & 0 & 1 & 0 & 0 & 1 & $*$ & $*$ & $*$ & $*$ & 1 & 0 & 0 & 1 \\
\hline
\end{tabular}

\section{Constructions on the Sets of State Transformations}

Task 1: Attainability

Let us return to composition of the state trajectory S2 $\rightarrow \mathrm{S} 3 \rightarrow \mathrm{S} 15 \rightarrow \mathrm{S} 8 \rightarrow \mathrm{S} 17$. When we ask if S17 is reachable from S2 then according to this composition the answer is NOT. However, they are other composition for which the answer is YES. A more general task is whether $S_{j}$ is achievable from $S_{i}$ ? And if it is not immediately visible, from the state transformation table, we must find at least one trajectory connecting $S_{i}$ with $S_{j}$. 
Another question that we deal with in partial algebra transforms is the problem of distance between states. Generally, the distance between states $S_{i}, S_{j}$ is given by the sequence of the transformations used. That is to say in the expression (13)

$$
\rho(\mathrm{S} 2, \mathrm{~S} 17)=(T 1, T 2, T 3, T 4) .
$$

This method is extremely disadvantageous because it is not possible to compare the distance even for trajectories based on the same states. For this reason, it is advisable to approximate the partial algebra on the set of states by some more symmetrical algebraic form.

\section{Task 2: Approximation of a partial algebra of state transformations by a cyclic group}

Cyclic groups can be found on the $X$ transformation set. There are many and there is a need to adopt a certain methodology for browsing the set of states. In our case, we have chosen the order of state indexes. We construct all cyclic groups whose state transforms begin in the state Si. However - only for some subsets of states can be done the following construction.

For the state S1 there are the following cyclic groups (for $k=\infty$ ) (according to Table 1):

$$
\begin{gathered}
T 1=(\mathrm{S} 1 \rightarrow \mathrm{S} 2) o(\mathrm{~S} 2 \rightarrow \mathrm{S} 1) . \text { Group } G 1(\mathrm{~S} 1)=\left\langle I, T 1, T 1^{2}, T 1^{3}, \ldots, T 1^{k}\right\rangle \text { is a free cyclic group, } \\
T 2=(\mathrm{S} 1 \rightarrow \mathrm{S} 11) o(\mathrm{~S} 11 \rightarrow \mathrm{S} 1) . \text { Group } G 2(\mathrm{~S} 1)=\left\langle I, T 2, T 2^{2}, T 2^{3}, \ldots, T 2^{k}\right\rangle \text { is a free cyclic group, }
\end{gathered}
$$

Further, e.g.:

$$
\begin{gathered}
T 7=(\mathrm{S} 1 \rightarrow \mathrm{S} 2) o(\mathrm{~S} 2 \rightarrow \mathrm{S} 9) o(\mathrm{~S} 9 \rightarrow \mathrm{S} 2) o(\mathrm{~S} 2 \rightarrow \mathrm{S} 1) . G 7(\mathrm{~S} 1)=\left\langle I, T 7, T 7^{2}, T 7^{3}, \ldots, T 7^{k}\right\rangle, \\
T 8=(\mathrm{S} 1 \rightarrow \mathrm{S} 11) o(\mathrm{~S} 11 \rightarrow \mathrm{S} 8) o(\mathrm{~S} 8 \rightarrow \mathrm{S} 16) o(\mathrm{~S} 16 \rightarrow \mathrm{S} 2) o(\mathrm{~S} 2 \rightarrow \mathrm{S} 1) . G 8(\mathrm{~S} 1)=\left\langle I, T 8, T 8^{2}, \ldots, T 8^{k}\right\rangle,
\end{gathered}
$$

Groups $G 7(\mathrm{~S} 1)$ and $G 8(\mathrm{~S} 1)$ are free cyclic groups.

By this way we form all cyclic groups that are parametrized by state S1 (i.e. they start in S1). Let us assume that we construct "m1" cyclic groups $G 1(\mathrm{~S} 1), \ldots, G m 1(\mathrm{~S} 1)$ for the state S1.

Now we form their direct product and we use theorem V3 (from Section 3). Direct product:

$$
D S(\mathrm{~S} 1)=G 1(\mathrm{~S} 1) \times G 2(\mathrm{~S} 1) \times \ldots \times G m 1(\mathrm{~S} 1) .
$$

According to theorem $\mathbf{V 3}$ is the group $G D P(\mathrm{~S} 1)$ constructed so that each of its elements is the product (one by one) of each group of the product $D S(\mathrm{~S} 1)$, is a cyclic group. This means that we can construct an artificial generator TDP (which is a function of generators of individual subgroups) - Figure 2 .

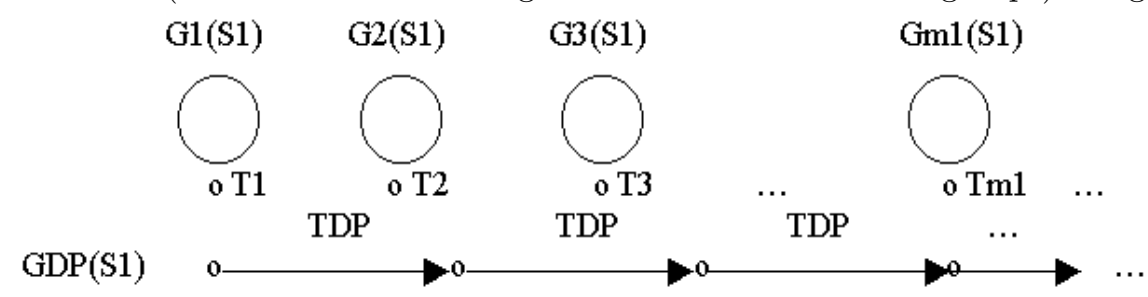

Figure 2: Approximation by cyclic group GDP(S1) with an artificial generator TDP

$$
G D P(\mathrm{~S} 1)=\left\langle I, T D P, T D P^{2}, \ldots, T D P^{k}\right\rangle .
$$

The TDP transformation is an artificial generator on the $G D P(\mathrm{~S} 1)$ group and its multiples can serve as the distance from the $S_{i}$ state to the selected $S_{k}$ state. (If the TDP generator can have its time length, we have built a chronometer on a set of states starting in S1.) Thus, if possible, we construct all cyclic groups by transforming $G D P(\mathrm{~S} 1), \ldots, G D P(\mathrm{~S} 19)$.

Task 3: Prediction of emergent situation on a complex system using a generator of cyclic group in the role of chronometer

We have already considered the possibility of EMS at work [11]. The usual objection was the number of selected states, perhaps large or rather small. For 100 states, by mathematics of matroids and by Ramsey Theory of Graph were derived that is required 111 additional PAES states. This was relatively high and it could be concluded that environmental systems are relatively stable to emergences. Considering that the selection of states is not absolute (and thus not their number), such a results were very rough.

The following calculation goes from the other side, from the contribution date of emergent phenomenon. At the same time, the elements of the matroid base considered as transformations on the cyclic group (from the 
previous paragraph) are calculated. This makes it possible to calculate the qualitative distance to the next emergence situation somewhat more precisely.

As an illustration of the application of the proposed method in a concrete example we introduce a process of extension of deserts in the Earth. The result of computation is the "time" of the next possible appearance of emergent situation. We use the following pilot data from the history: a) Global warming: before 11700 years (from now), [19] is considered global warming as one of the substantial Emergences. b) The land drying began 6000 years ago [17, 18]. This was a long-term average trend, with intermittently alternating dry and wet seasons. 2006:

Out of all the available data, we use for our computation only a single reliable fact that was published in

"Deserts cover 47 million km2 of dry land. This amounts to $35 \%$ of the land area of the world."

(We will speak about a single world desert.)

We denote the area of the desert (DA) in time tD as DA $\left(t_{D}\right)$. We consider two-times $t_{D}: 2006$ and time "No Desert (ND)". In order to conserve the logic of modeling the development of the desert (as a single desert of the world) we introduce the following variable $s=1+\mathrm{DA}\left(t_{D}\right)$ and for variables $s_{\text {em }}$ and $s_{n o m}$ :

$$
\begin{gathered}
s_{\text {em }}=1+\mathrm{DA}(2006)=1+0.35=1.35 \\
s_{\text {nom }}=1+\mathrm{DA}(\mathrm{ND})=1+0=1
\end{gathered}
$$

If we consider that at the beginning of some paleoecological cycle there was no dry land, then the corresponding contribution to the Power of the emergent phenomenon related to year 2006 is

$$
\Delta H_{p}(\boldsymbol{B}+\mathbf{1})=\sum_{i=1}^{1}\left(\omega_{i}\left(s_{\text {iem }} / s_{\text {inom }}\right)^{2}\right)^{1 / 2}=\left(s_{\text {em }} / s_{\text {nom }}\right)=(1.35 / 1)=1.35
$$

(Only for checking the logic of the computation: for time $t_{D}=$ ND we have $H_{p}(\boldsymbol{B}+\mathbf{1})=1$.)

Considering the emergent phenomenon only as a consequence of "activities" of desert (and not of other influences, e.g., some anthropogenic activities of human) and using quotient $(u / c)=0.1$ (tuned in $[6,7]$ we compute from (8))

$\# \boldsymbol{B}=14(13.5)$ and $R(3,14)=\# X \in[66,78]$, using the table in [15]. The next Ramsey number is $R(3,15)=\# X \in[73,78]$, from [15]. Continuing with [15] we compute the nearest Ramsey numbers.

The nearest Ramsey numbers for $\# \boldsymbol{B}=14$ are

$$
R(3,14)=[66,78], R(4,14)=[141,349], R(5,14) \geq 221 .
$$

The nearest Ramsey numbers for $\# \boldsymbol{B}=15$ are

$$
R(3,15)=[73,78], R(4,15)=[153,417], R(5,15) \geq 242 .
$$

Combinations of Ramsey numbers for the selection of a minimal difference

$$
\begin{aligned}
& \Delta f(R N)=R N(x, 15)-R N(y, 14): \\
& R N(y, 14):(66,78,141,349,221) \\
& R N(y, 15):(73,78,153,417,242)
\end{aligned}
$$

and we express $((R N(x, 15)-R N(y, 14) / \Delta f(R N))$ in the sequence

$$
\text { (73-66/7), (78-66/12), (78-78/0), (153-141/13). }
$$

Note 9.1: The next differences $\Delta f(R N)$ are larger than the introduced differences and will not be considered.

For predicting PAES (10), we use only two differences from the sequence above: $\Delta f(R N)=7$ and $\Delta f(R N)=0$ :

$$
I F(\# E 1 \geq 7) \text { OR }(\# E 1=0) \Rightarrow(P A E S) .
$$

Using the length of transform $T D P(. .$.$) from Task 2, as one year, we may estimate the time for the$ appearance of the next emergent situation. Thus, we have for $i=1,2$ equations

$$
\begin{gathered}
\Delta f(R N)_{i} / R N=\Delta t(\text { NextPAES }) / t(\text { PreviousPAES }) \\
\Delta t(\text { NextPAES })=\left(\Delta f(R N)_{i} / R N\right) t(\text { PreviousPAES })
\end{gathered}
$$


For $\Delta f(R N)_{1}=7, R N=66$, and for $t$ (PreviousPAES) $=6000$ years back (from 2006), (this is the time of the beginning of drying of the land and the development of deserts) we compute $\Delta t$ (NextPAES) $=636$ (in future) from 2006. For the case $\Delta f(R N)_{2}=0, \Delta t$ (NextPAES) $=0$ - which means the year 2006!

The results for $\Delta t$ (NextPAES) represent a qualitative distance (large/small) from time 2006. This means that the computed numbers very roughly estimate the time for the next Possible Appearance of an Emergent Situation (with regard to development of deserts). (With a great sorry we remind a horrible drought in Central Europe in 2018!!)

\section{Conclusion}

Building on the findings reported in $[6,7]$, in this paper we have applied the method for prediction of the appearance of emergent situations in complex systems. From a technical point of view, the paper combines the essentials from universal partial algebras, the Theory of Groups, the Theory of Matroids and Ramsey graph theory (RGT). The paper uses a qualitative modeling approach (supported by expert guidance) that enables the description of a very complicated complex system to be reduced by deploying expert knowledge.

Acknowledgement: This research has been supported by means of SGS17/P2301/OHK2-015. This support is very gratefully acknowledged.

\section{References}

[1] Kinsner, W. 2008. Complexity and its Measures in Cognitive and other Complex Systems. In ICCI08. Stanford, CA, USA, pp. 2-75.

[2] Bila, J., Pokorny, J., and Bukovsky, I. 2011. Qualitative Modeling and Monitoring of Selected Ecosystem Functions. Ecological Modeling 222, pp. 3640-3650.

[3] Rodriguez, R., Mexicano, A., Bila, J., Cervantes, S., and Ponce, R. 2015. Feature Extraction of Electrocardiogram Signals by Applying Adaptive Threshold and Principal Component Analysis. Journal of Applied and Research Technology 13, 4, pp. 71-80.

[4] Monasterio, V., Laguna, P., and Martínez, J. P. 2009. Multilead Analysis of T-Wave Alternants in the ECG using Principal Component Analysis. IEEE Trans. Biomed. Eng. 56, 7, pp. 1880-1890.

[5] Bila, J. 2018. Capacity of the Space of Properties. MENDEL 24, pp. 39-46.

[6] Bila, J. 2017. Emergent Phenomena in Complex Systems and their detection. International Journal of Enhanced Research in Science Technology and Engineering 6, 12, pp. 40-53.

[7] Bila, J. 2014. Emergent phenomena in Natural Complex Systems. In Proc. of Interdisciplinary Symposium of Complex Systems. Springer, Heidelberg, Germany, pp. 89-100.

[8] Petty, M. D. 2014. Modeling and validation challenges for Complex Systems. M S Journal 9, 1, pp. 25-35.

[9] Grätzer, G. 1968. Universal Algebra. Van Nostrand Co., Princeton, USA.

[10] Grenander, U. 1981. Regular Structures. Lectures in Pattern Theory, Vol. 3. Springer-Verlag, Berlin, Germany.

[11] Bila, J., Novak, M., and Vrba, J. 2016. Detection of emergent situations in ecosystems. MATEC Web of Conferences 76, pp. 1-6.

[12] Saaty, T. L. 1978. Exploring the Inference between Hierarchies, Multiple Objectives and Fuzzy Sets. Fuzzy Sets and Systems 1, 1, pp. 57-68.

[13] Oxley, J. G. 2001. Matroid Theory. Oxford Science Publications, Reprinted Edition, UK.

[14] Ramsey, F. P. 1930. On a Problem of Formal Logic. Proc. London Math. Soc 30, pp. 264-286.

[15] Weinstein, W. 2019. Ramsey Number. A Wolfram Web Resource [online, accessed 19.06.2019] http://mathworld.wolfram.com/RamseyNumber.html

[16] Langford, G. O. 2018. Phenomenological and Ontological models for Predicting Emergence. In Engineering Emergence: Modeling and Simulation Approach. CRS Press/Taylor and Francis, Boca Raton, USA, pp.119-160.

[17] Alverson, K. D., Bradley, R. S., and Pedersen, T. F. 2003. Paleoclimate, Global Change and the Future. Springer-Verlag: Berlin, Heidelberg, New York.

[18] Bradley, R. S., Briffa, K. R., Cole, J., Hughes, M. K., and Osborn, T. J. 2003. The climate of the last millennium. In Paleoclimate, global change and the future. Springer - Berlin, Heidelberg, pp. 105-141.

[19] Bond, G., Showers, W., Cheseby, M., Lofti, R., Almasi, P., deMenocal, P.B., Priore, P., Cullen, H., Hajdas, I., and Bonani, G. 1997. A pervasive millennial-scale cycle in the North Atlantic Holocene and glacial climates. Science 294, pp. 2130-2136. 